\title{
Optimizing Dead Mileage in Urban Bus Routes. Dakar Dem Dikk Case Study
}

\author{
Cheikh B. Djiba ${ }^{1}$, Mamadou Balde ${ }^{1}$, Babacar M. Ndiaye ${ }^{2}$, Roger M. Faye ${ }^{1}$, Diaraf Seck ${ }^{3}$ \\ ${ }^{1}$ Laboratory of Information Processing, ESP Dakar, Cheikh Anta Diop University, Dakar, Senegal \\ ${ }^{2,3}$ Laboratory of Mathematics of Decision and Numerical Analysis, FASEG, \\ Cheikh Anta Diop University, Dakar, Senegal \\ ${ }^{3}$ Unité Mixte Internationale, UMMISCO, Institut de Recherche Pour le Développement, Bondy, France \\ Email: cdjiba83@yahoo.fr, mbalde111@ucad.sn, \{roger.faye, babacarm.ndiaye_diaraf.seck\}@ucad.edu.sn
}

Received April 16, 2012; revised May 19, 2012; accepted June 2, 2012

\begin{abstract}
This paper studies the buses assignment from their depots to their routes starting points in urban transportation network. It describes a computational study to solve the dead mileage minimization to optimality. The objective of this work is to assign the buses to depots while optimizing dead mileage associated with pull-out trips and pull-in trips. To do so, a new mixed-integer programming model with 0 - 1 variables is proposed which takes into account the specificity of the buses of Dakar Dem Dikk (the main public transportation company in Dakar). This company manages a fleet of buses some of which, depending on road conditions cannot run on part of the network. Thus, buses are classified into two categories and are assigned based on these categories. The related mixed-integer 0 - 1 linear program is solved efficiently to minimize the cumulative distance covered by all buses. Numerical simulations on real datasets are presented.
\end{abstract}

Keywords: Global Optimization; Dead Mileage; Assignment; Urban Transportation

\section{Introduction}

Dakar Dem Dikk (3D) is the main public urban transportation company in Dakar. It manages a fleet of buses with different technical characteristics so that a great part of the bus fleet cannot have access to all the network. Buses are parked overnight at Ouakam and Thiaroye depots (see Figure 1). Starting points of routes (or terminals) are different from depots. A bus has to cover the distance from its depot to the starting point of its route before being undertaken on a regular service. This distance is known as "dead mileage" or "dead heading" because no service is provided while covering it.

The objective is to minimize the cumulative distance covered by all the buses from their depots to the starting points of their routes and vice versa. End points of the routes are taken to be the same as the starting points.

In addition, depending on roads conditions and technical characteristics of buses, some of them cannot circulate on some roads of the network. The capacities of the respective depots and the number of buses are known.

More accurately, the 3D urban transportation network can be defined by the valued graph $G=(N, A, C)$ (see Figures 1 and 2, where all depots, terminals, bus stops and routes (158 for Ouakam depot, and 131 for Thiaroye depot) are represented:
- $\quad N$ is the set of nodes which represents depots, terminals (starting points) or bus stops.

- A is the set of arcs in $N \times N$, representing the set of routes connecting terminals and depots, terminals and bus stops or two bus stops.

- $\mathrm{C}$ is the set of distances between nodes.

- There are both good and bad routes, that is to say there exist pairs $(i, j) \in A$ such that the route connecting $i$ and $j$ is in a bad condition.

A workday for a given bus is defined as a sequence of trips, dead mileage, parking stops and pull-out/pull-in trips from/to the assigned depot. Given the diversity of transportation modes in Dakar and the high customer demand (in terms of service quality and network coverage), 3D decision makers must manage multiple resources: buses, employees, depots, etc. They make several important decisions such as network design, timetabling, vehicle scheduling, construction of new depots, etc. The 3D company focuses on efficient use of resources, especially buses and drivers. Decision markers here play a crucial role. However, two important aspects are taken into account for the determination of frequencies and schedules: facilities supply and transport demand.

In practice, due to their complexity, planning problems are often divided into three levels of decisions which are solved sequentially, see Boctor et al. [1]: 


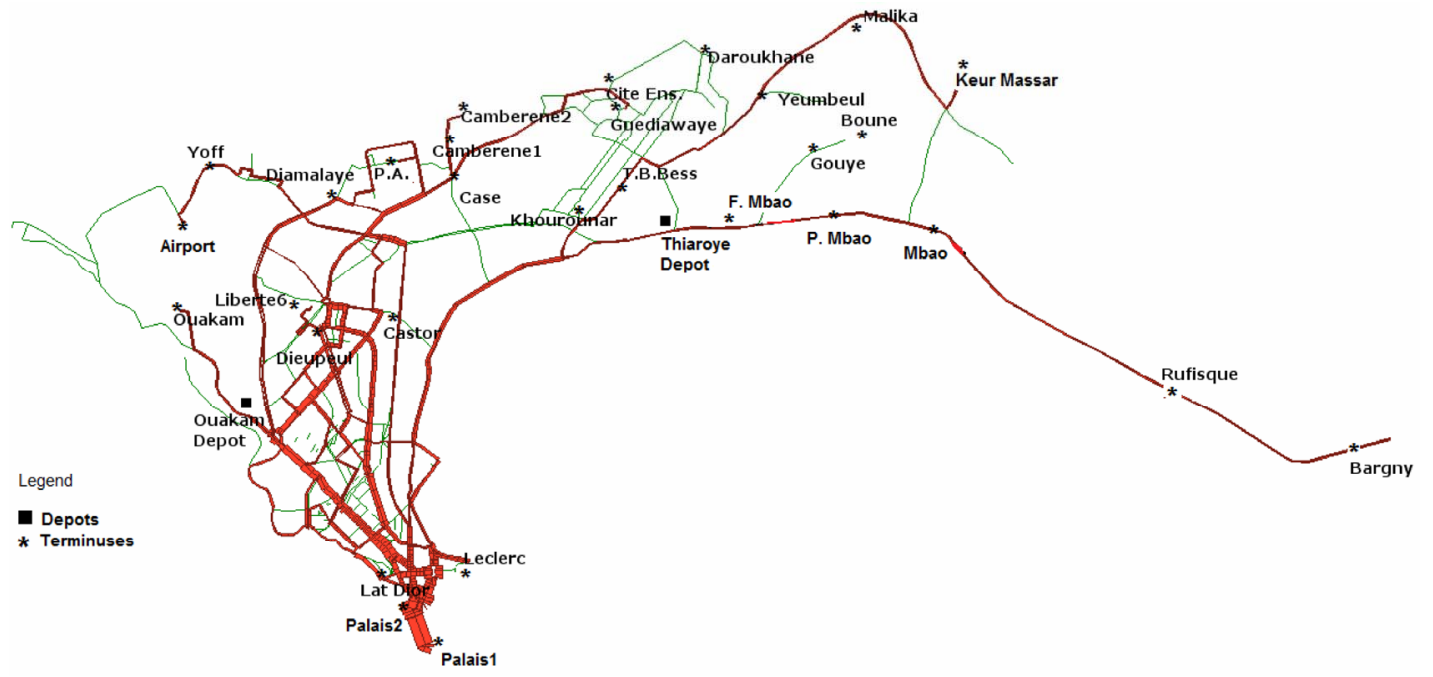

Figure 1. 3D transportation network.

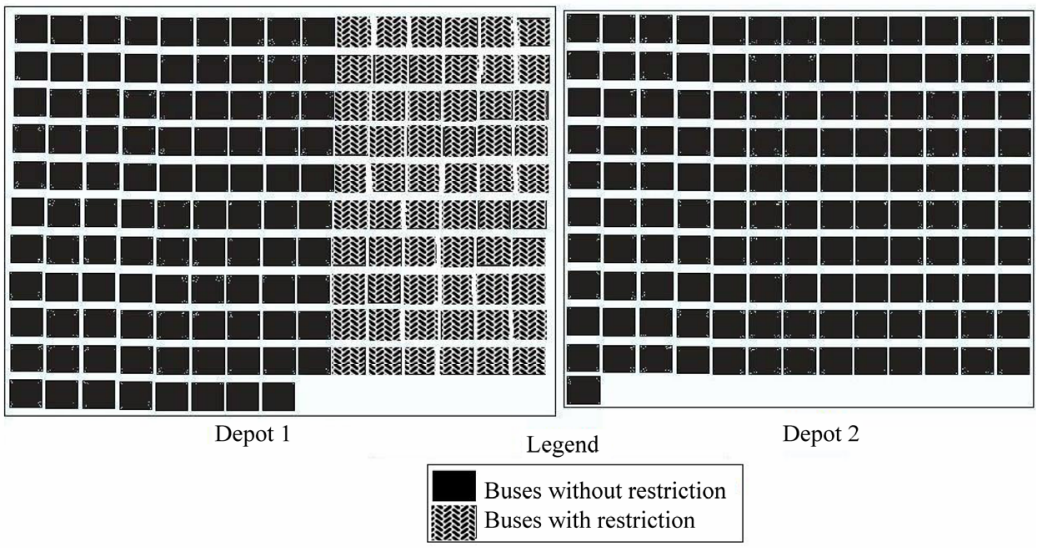

Figure 2. Depots with different bus types.

1) The strategic planning level involving long-term decisions: network design, bus stop problem, etc.

2) The tactical planning level that concerns mean-term decisions: trip frequency scheduling, timetabling, etc.

3) The operational planning level concerning the short-term decisions and deals with the current transportation operations: vehicle scheduling, driver scheduling, etc.

The inevitable problems related to current operations can be addressed through adjustments in timetables. In the past, there were no problems related to dead mileage that warrant serious attention. Even if they cropped up they were tackled by applying some techniques based on commonsense and experience. Nowadays, due to the fact that most of the urban transportation companies have large fleets of buses, bus stops and terminals, problems related to dead mileage have become so complex that 3D's rudimentary techniques seem unsuitable. Thus, the need to use analytical tools to deal with urban transportation system problems has called the attention of 3D decision makers. Since dead mileage trips create an additional cost factor, the minimization of total distance covered by all the buses will reduce this cost. Thus, the minimization of this cost is an important optimization goal.

The strategic level decision problem of assigning buses to depots while optimizing dead mileage, is known to be a NP-Hard problem, see e.g. Boctor et al. [1]; Hsu [2]; Prakash et al. [3]. Several researchers have employed analytical tools to deal with urban transportation system problems in the recent past. Sharma and Prakash [4] have applied analytical tools for solving a prioritized bicriterion dead mileage problem related to an urban bus transportation system. The problem is to determine an optimal number of buses to be parked overnight at respective depots and an optimal schedule to take buses from the depots to the starting points of their routes. The capacities of the respective depots and the number of buses required at the starting points of routes are known. The minimization of the cumulative distance covered by 
all the buses and that of the maximum distance between the distances covered by individual buses from the depots to the starting points of their routes are the first and second priority objectives, respectively. In Hsu [2], Hsu has extended Sharma and Prakash's problem by introducing an additional all-or-nothing constraint which stipulates that all the buses plying on a route must come from the same depot. In Prakash et al. [3], they developed an algorithm to obtain the set of nondominated solutions of a two-objective problem. The two objectives were to minimize the cumulative distance covered by all the buses and the maximum distance among the distances covered by individual buses from the depots to the starting points of their respective routes. Kasana and Kumar [5] proposed an extension that makes it possible to treat p objectives and take up the data in Prakash et al. [3]. It should be noted that these contributions take into account the capacity of depots in a period corresponding to a workday; and also buses should leave and return to the same depot. In Agrawal and Dhingra [6], a singleobjective dead mileage problem is considered. The problem is to determine optimal program aiming at increasing the depots capacities and the number of buses to be parked overnight at respective depots with a schedule to take the buses from the depots to the starting points of their routes.

Prakash and Saini [7] have considered a bi-criterion dead mileage problem: the problem is to select an optimal site for a new depot of specified capacity from among several potential sites. An optimal schedule to take buses from the depots to the starting points of their routes and the spare capacity available at each of the depots after the construction of the new depot are also determined. Moreover, Pepin et al. [8] developed a heuristic approach for the vehicle scheduling problem with multiple depots. The objective is to determine schedules of minimum cost for vehicles assigned to depots. In their work, Boctor et al. [1] studied the problem of minimizing the dead mileage, with two models, in order to select a new depot among several potential sites. Their first model gives the buses assignment by requiring that all buses must return to the same depot. The model takes into account the storage capacity by dividing the day into $T$ periods. In their second model, a bus can end its route to a depot which is different from its initial one. However, neither the bus types nor the road condition are integrated in this model. In its case, 3D makes its planning and takes these two constraints into account. Sometimes it happens that the decision maker is not able to assign priorities to both bus types and road conditions. In such a situation, help can be provided to the decision makers by presenting them with a set of solutions.

In the present paper, an attempt is made to deal with this type of situation in the context of dead mileage. We propose a mixed-integer programming problem with 0 1 variables to deal with the dead mileage minimization problem of 3D. The new model obtained is based on the second one developed in Boctor et al. [1]. The model assigns each pull-in trip and each pull-out trip to a depot so as to minimize the mileage associated. It takes into account both the bus types and the network condition $[9,10]$, and we show that this technique is of practical importance. With some processing techniques, we solve instances of $3 \mathrm{D}$ which could not be solved by their rudimentary techniques. By dividing the day into $T$ periods, this model is applied to 3D's scenarios. We obtain through numerical simulations (by using the commercial MILP solver, namely IBM-CPLEX [11]) the dead mileage reduction.

This paper is organized as follows: In Section 2, we present the model that minimizes the mileage associated with the pull-out trips and the pull-in trips. Computational analyzes using IBM-CPLEX V12.3 and the frequency assignment of buses are carried out in Section 3. Finally, summary and conclusions are presented in Section 4.

\section{Definition of the Dead Mileage Optimization Problem}

The problem can be stated as follows. Given

- the bus types,

- a set of depots,

- a set of routes,

- and a set of terminals;

assign buses so as to minimize dead mileage traveled by buses, for their pull-out trips and their pull-in trips. The dead mileage corresponds to a route where no service is provided while covering this distance.

The following assumptions are introduced to formulate the dead mileage problem:

- A certain number of routes are assigned to each depot, and consequently a certain number of buses.

- Each depot has a capacity.

- There are several bus types divided into $r$ categories, with $K=\{1,2, \cdots, r\}$ the set of bus types.

- All buses on which there is a restriction (belong to $K_{1}$ ) come from the same depot.

- Taking into account the capacities of depots, bus types $k \notin K_{1}$ can return to the nearest depot ( $K_{1}$ is the set of bus restrictions).

- A bus is said to be compatible with a depot if at the end of its route, it can return to this depot.

The following notations are used to describe the procedures for optimizing dead mileage.

\subsection{Indices-Parameters-Sets}

$i$ : depots $(i=1, \cdots, m)$. 
$j:$ routes $(j=1, \cdots, p, p+1, \cdots, n)$.

$t$ : periods $(t=1, \cdots, T)$.

$k$ : bus types, $k \in K$.

$p$ : number of bus types with restrictions $\left(p=\left|K_{1}\right|\right)$.

$e_{i j}$ : dead mileage associated with the pull-out trip of route $j$ from depot $i$.

$f_{i j}$ : dead mileage associated with the pull-in trip of route $j$ to depot $i$.

$c_{i}$ : capacity of depot $i$.

$M$ : total number of available buses for the $m$ depots.

$S_{i t}$ : number of buses leaving from depot $i$ at period $t$.

$R_{i t}$ : number of buses going to depot $i$ at period $t$.

$K$ : the set of bus types, $K=\{1,2, \cdots, r\}$.

$K_{1}$ : the set of bus types with restrictions.

\subsection{Decision Variables}

$x_{i j}$ : binary variable which becomes 1 if the pull-out trip of route $j$ is associated with depot $i, 0$ otherwise.

$y_{i j k}$ : binary variable which becomes 1 if the pull-in trip of route $j$ is associated with depot $i$ for the bus type $k$ (the bus which performs the route is compatible to this depot), 0 otherwise.

$n_{i t}$ : number of buses in depots $i$ at the beginning of period $t$.

The dead mileage problem may be formulated as follows:

$$
\min \sum_{i=1}^{m} \sum_{j=1}^{n} e_{i j} x_{i j}+\sum_{k \in K} \sum_{i=1}^{m} \sum_{j=1}^{n} f_{i j} y_{i j k}
$$

s.t.

$$
\begin{gathered}
\sum_{i=1}^{m} x_{i j}=1 \quad \forall j=1, \cdots, n \\
\sum_{i=1}^{m} y_{i j k}=1 \quad \forall j=1, \cdots, n \quad \forall k \in K \\
\sum_{k \in K} \sum_{i=1}^{m} \sum_{j=1}^{n} y_{i j k}=p \\
n_{i t}=n_{i t-1}-\sum_{j \in S_{i t-1}} x_{i j}+\sum_{j \in R_{i t-1}} y_{i j k} \\
\forall i=1, \cdots, m \quad \forall t=2, \cdots, T \quad \forall k \in K \\
n_{i t} \leq c_{i} \quad \forall i=1, \cdots, m \quad \forall t=1, \cdots, T \\
\sum_{i=1}^{m} n_{i 1}=M \quad \forall \quad \forall i=1, \cdots, m \\
n_{i 1}=n_{i T+1} \quad \forall i=1, \cdots, m \quad \forall j=1, \cdots, n \\
x_{i j} \in\{0,1\} \quad \forall i=1, \cdots, n \quad \forall k \in K \\
y_{i j k} \in\{0,1\} \quad \forall i=1, \cdots, m \quad \forall j=1, \cdots, T \\
n_{i t} \geq 0 \quad \forall i=1, \cdots, m \quad \forall t=1
\end{gathered}
$$

The objective function (1) minimizes the mileage asso- ciated with the pull-out trips and pull-in trips while taking into account the bus that covers the route. The departure depots may be different from the return ones. It is a linear objective function. Constraint (2) implies that the pull-out trip of a route is associated with a single depot. Constraint (3) ensures that the pull-in trip is also associated with a single depot and takes into account the bus compatibility. Constraint (4) is effective only when $y_{i j k}$ equals one. It restricts the number of $k$ type bus pull-in trips to exactly $p$, where $\mathrm{p}$ is the number of bus types with restrictions. Constraint (5) makes it possible to have, in real time, the number of buses at each depot. Constraint (6) implies that the number of buses in the depot $i$, at each period, is less than or equal to the capacity of this depot. Constraint (7) ensures that the number of buses in all depots is equal to the number of available buses. Constraint (8) ensures the feasibility of the model. The number of buses in the morning (starting period) must be equal to the number of buses in the evening (end period). Constraints (9), (10) and (11) represent domains of the variables.

\section{Numerical Experiments}

This section shows how the problem is applied to real data of 3D. Using the model, the operational costs resulting from the reduction of dead mileage in urban buses can be calculated.

Dead mileage does not only have an impact on revenue but also in increases of fuel consumption, operational costs, pollution etc. As all these bad consequences could be reduced with dead mileage, it is thus an obligation to optimize it.

The operational costs of dead mileage, by a vehicle per kilometer, is evaluated jointly with 3D. Considering that the bus average speed is 60 kilometer per hour, the cost of vehicle use is 1000 FCFA ( $\simeq 1.5$ euro) per kilometer.

Located at km 4.5 avenue of Cheikh Anta Diop, the 3D company manages a fleet of 410 vehicles using two depots: a depot located in Ouakam (km 4.5 Cheikh Anta Diop avenue, the head office of the company) and a depot located in Thiaroye (suburbs of Dakar, 19 kilometers far from Dakar city).

To ensure network coverage, 3D manages its services by using 17 lines (see Tables 1 and 2, with 11 from Ouakam depot and 6 from Thiaroye depot. Each line ensures a certain number of routes. Presently, the total number of routes in the network is 289. Thirty (30) permanent terminals and 810 bus stops are used (see Figure 1, where bus stops are not representeddue to their quantity). The maps in Figures $\mathbf{1}$ and $\mathbf{3}$ are obtained by using the software EMME [12]. A daywork is divided into 38 periods of 30 minutes. The first route of the day should arrive at its terminal starting at 6:00 am. Given the 


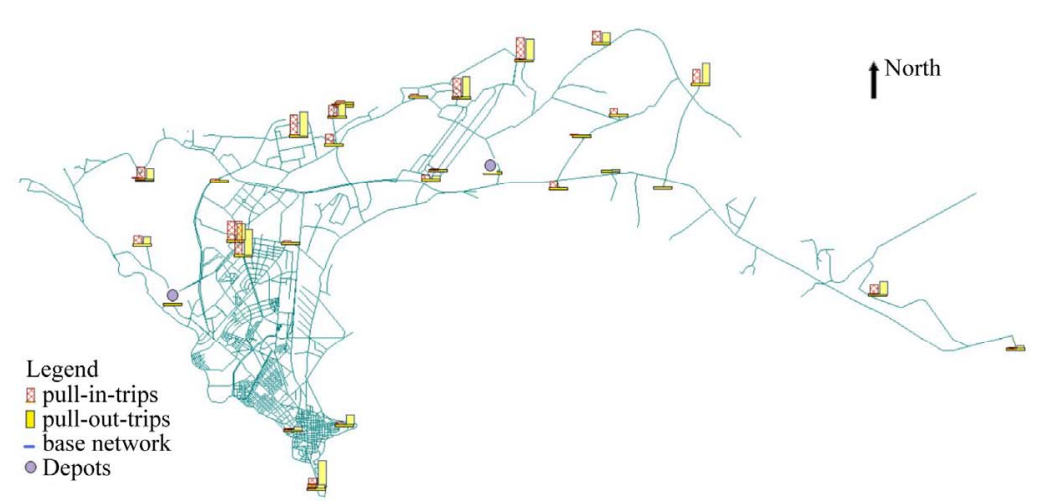

Figure 3. New pull-in/pull-out trips given by the model.

Table 1. Line assignments from Ouakam depot.

\begin{tabular}{llllllllllll}
\hline \multicolumn{11}{c}{ Ouakam depot } \\
\hline Lines & 1 & 4 & 6 & 7 & 8 & 9 & 10 & 13 & 18 & 20 & 23 \\
\hline
\end{tabular}

Table 2. Line assignments from Thiaroye depot.

\begin{tabular}{ccccccc}
\hline \multicolumn{7}{c}{ Thiaroye depot } \\
\hline Lines & 2 & 5 & 11 & 12 & 15 & 16 \\
\hline
\end{tabular}

distance between depot and this terminal, the first vehicle must leave the current depot at 5:45 am. Therefore, the first period of the model begins at 5:30 am. The first and the last periods correspond to 05:30 am - 06:00 am and 11:30 pm - 00:00 am, respectively.

The input data needed to use the model are:

- Current number of depots, $m=2$ (Ouakam and Thiaroye (see Figure 1));

- Total number of routes, $n=289$;

- Number of routes associated with Ouakam depot: 158 (see Figure 2);

- Number of routes associated with Thiaroye depot: 131 (see Figure 2);

- Set of bus types: $\{1,2\}$;

- Set of bus types in which there is a restriction: $\{2\}$;

- Number of periods $T$ : 38 ;

- Number of bus type 1: 229;

- Number of bus type 2: 60 (number of buses on which there is a restriction);

- Number of terminals (starting points): 30 (see Figure 1).

Relevant informations such as a storage plan of the depots and time interval in which each bus is available (for pull-out trips and pull-in trips), are usually given. In addition, the following information is known in advance:

- capacities of Thiaroye and Ouakam depots,

- and distances between depots and end points of routes.

Tables $\mathbf{3}$ and $\mathbf{4}$ give the total number of buses to be parked overnight and the number of buses to be sent from depots to the starting points of routes. Recall that, all scenarios are given by 3D.

To represent the pull-out trips and the pull-in trips, we mentioned only the periods where there are actually pullin trips and pull-out trips of routes. In periods 8 to 27, which correspond respectively to the intervals from 08:30 am - 09:00 am to 06:00 pm - 06:30 pm where no entry is made. That is why they are not represented in tables.

Tables $\mathbf{3}$ and $\mathbf{4}$ show the number of pull-out trips and pull-in trips for all routes on the network, and Figure 1 illustrates the locations of Ouakam and Thiaroye depots and the starting points. When we look at this map, we can notice that there are sites where the demand is high. The cumulative distance (for the 17 lines) covered by all the buses from the depots to the starting of their routes and from the end points back to their depots is 7881.9 kilometers. Therefore, the daily cost is $7,881,900$ FCFA ( $\simeq 11,822.85$ euro) for the 38 periods (from $05: 30$ am to $00: 00 \mathrm{am})$. As the 38 periods correspond to a workday, the total cost is $39,409,500$ FCFA ( $\simeq 59,114.25$ euro) for 5 workdays (from Monday to Friday). Therefore, the total cost is $157,638,000$ FCFA ( $~ 236,457$ euro) for a month (are counted only workdays).

Recall that, our proposed model takes into account both the bus types and the network condition. The numerical experiments were executed on a computer: $2 \times$ Intel(R) Core(TM)2 Duo CPU $2.00 \mathrm{GHz}, 4.0 \mathrm{~Gb}$ of RAM, under UNIX system.

The numerical tests were performed by using the IBMCPLEX's MILP solver [11], with a total number of 1114 variables, where the number of binary variables is 1036 . The solution obtained is an optimal one (of this problem) wherein the priority is assigned to the minimization of the dead mileage.

The total distance covered by all the buses from depots to starting points of routes and from the end points back to their depots is 5268.8 kilometers. Therefore, a total cost of 5,268,800 FCFA ( $\simeq 7903.2$ euro) for a workday.

The total cost is $26,344,000$ FCFA ( $\simeq 39,516$ euro) for 5 workdays. Therefore, a total cost of 105,376,000 FCFA 
Table 3. Current situation of pull-out trips.

\begin{tabular}{ccccccccc}
\hline Periods & 1 & 2 & 3 & 4 & 5 & 6 & 7 & Total \\
\hline Thiaroye pull-out trips & 3 & 25 & 40 & 34 & 19 & 8 & 2 & 131 \\
Ouakam pull-out trips & 0 & 20 & 37 & 55 & 35 & 9 & 2 & 158 \\
\hline
\end{tabular}

Table 4. Current situation of pull-in trips.

\begin{tabular}{cccccccccccc}
\hline Periods & 28 & 29 & 30 & 31 & 32 & 33 & 34 & 35 & 36 & 37 & 38 \\
\hline $\begin{array}{c}\text { Thiaroye } \\
\text { pull-in } \\
\text { trips }\end{array}$ & 2 & 2 & 11 & 8 & 26 & 14 & 21 & 26 & 13 & 7 & 1 \\
$\begin{array}{c}\text { Ouakam } \\
\text { pull-in } \\
\text { trips }\end{array}$ & 0 & 5 & 4 & 12 & 30 & 41 & 40 & 22 & 3 & 1 & 0 \\
\hline
\end{tabular}

( $\simeq 158,064$ euro) is obtained for a month.

So, with our model we obtain a saving of 52,262,000 FCFA ( $\simeq 78,393$ euro) monthly, see Table $\mathbf{5}$ where distances are in kilometers.

Tables 6 and 7 show the pull-out trips and the pull-in trips, respectively. First, some modifications in the pullout trips are pointed out. With the assignment of 3D we have 131 and 158 buses assigned to Thiaroye and Ouakam depots, respectively. From our numerical experiments we obtain 137 and 152 buses assigned to Thiaroye and Ouakam depots, respectively. Therefore, 6 routes are reduced from Ouakam depot and assigned to Thiaroye. But, the main difference appears in the assignments by period.

Indeed, for the first period: the 3 Thiaroye pull-out trips, corresponding to the starting points Parcelles Assainies, Case and Palais 1 are assigned to Ouakam depot. During period 2, Ouakam depot loses 2 pull-out trips to the benefit of Thiaroye depot which has 27. The same scenario occurs in periods 3 and 4 where, respectively, 4 and 31 additional routes are assigned to Thiaroye depot. On the other hand, 18, 8 and 2 routes are moved from Thiaroye depot and assigned to Ouakam depot, for the periods 5, 6 and 7, respectively.

For the pull-in trips, there is no change in the $28^{\text {th }}$ period. From period 29 to 34, we have respectively 3, 2, 1, 7, 20 and 20 additional routes; assigned to Thiaroye depot. From period 35 to 38, we obtain respectively 26, 13, 7 and 1 additional routes, assigned to Ouakam depot.

Figure 3 illustrates the new pull-in and pull-out trips at all terminals except depots. At each terminal are represented the cumulative pull-in and pull-out trips. Trips from/to depots are ignored in this figure. One can notice that the amounts of trips are higher in terminals located in the northern side; the main reason for this fact is that most of 3D costumers leave suburbs. So during the morning 3D manages to assign an important quantity of buses to the suburbs' terminal to carry people to offices, factories, firms that are downtown. In the evening the re-
Table 5. Comparison of distances and costs.

\begin{tabular}{ccccc}
\hline $\begin{array}{c}\text { Cumulative } \\
\text { distance }(\mathrm{km})\end{array}$ & $\begin{array}{c}\text { Current cost } \\
(\text { FCFA })\end{array}$ & $\begin{array}{c}\text { Actual distance } \\
(\mathrm{km})\end{array}$ & $\begin{array}{c}\text { Actual cost } \\
(\text { FCFA })\end{array}$ & $\begin{array}{c}\text { Savings } \\
(\text { FCFA) }\end{array}$ \\
\hline 7881.9 & $157,638,000$ & 5268.8 & $105,376,000$ & $52,262,000$ \\
\hline
\end{tabular}

Table 6. New situation of pull-out trips by using the model.

\begin{tabular}{ccccccccc}
\hline Periods & 1 & 2 & 3 & 4 & 5 & 6 & 7 & Total \\
\hline Thiaroye pull-out trips & 0 & 27 & 44 & 65 & 1 & 0 & 0 & 137 \\
Ouakam pull-out trips & 3 & 18 & 33 & 24 & 53 & 17 & 4 & 152 \\
\hline
\end{tabular}

Table 7. New situation of pull-in trips by using the model.

\begin{tabular}{cccccccccccc}
\hline Periods & 28 & 29 & 30 & 31 & 32 & 33 & 34 & 35 & 36 & 37 & 38 \\
\hline $\begin{array}{c}\text { Thiaroye } \\
\text { pull-in } \\
\text { trips }\end{array}$ & 2 & 5 & 13 & 9 & 33 & 34 & 41 & 0 & 0 & 0 & 0 \\
$\begin{array}{c}\text { Ouakam } \\
\text { pull-in } \\
\text { trips }\end{array}$ & 0 & 2 & 2 & 11 & 23 & 21 & 20 & 48 & 16 & 8 & 1 \\
\hline
\end{tabular}

versed phenomenon takes place.

Sites where demand is high correspond to starting points of Dieupeul, Liberte 6, Palais 1, Guediawaye, Camberene 1, Daroukhane and Keur Massar. The starting points of Airport, Camberene 2, Mbao and Malika are the second category of concentration. The results show that by allowing buses to return to their starting points or depots different from the starting ones, we can significantly reduce the dead mileage, and thus obtain greater savings. All this database is validated by $3 \mathrm{D}$ officials.

\section{Concluding Remarks}

In this paper, we described how the new model of optimizing dead mileage can be used to compute an optimal solution of the 3D urban transportation problem. We developed a mixed-integer programming problem with 0 - 1 variables which takes into account road network and achieved greater savings. The results showed that 3D can significantly reduce the cumulative distance covered by all the buses from their depots to the starting points of their routes and also from starting points of their routes back to their depots. To be more competitive, alterations, roads repairs, fleet renewal and constructions of new depots will improve the results performance. Furthermore, the model considered in this work can be used in the case where the end points of routes are different from starting points.

\section{Acknowledgements}

We would like to thank all DSI's (Division Système d'Information) members of 3D for their time and efforts in providing us with significant improvements of this paper. 


\section{REFERENCES}

[1] F. F. Boctor, J. Renaud and S. Bournival, "Choix de Sites D’Entrepôts Pour Les Autobus de Transport Urbain: Le Cas du Réseau de Transport de La Capitale,” 2006. http://www.fsa.ulaval.ca/personnel/renaudj/pdf/Recherch e/RTC\%20INFOR.pdf

[2] J. A. Hsu, "Discussion on the Paper: Optimizing Dead Mileage in Urban Bus Routes,” Journal of Transportation Engineering, Vol. 114, No. 1, 1988, pp. 123-125. doi:10.1061/(ASCE)0733-947X(1988)114:1(123)

[3] S. Prakash, B. V. Balaji and D. Tuteja, "Optimizing Dead Mileage in Urban Bus Routes through a Nondominated Solution Approach,” European Journal of Operational Research, Vol. 114, No. 3, 1999, pp. 465-473. doi:10.1016/S0377-2217(98)00238-0

[4] V. Sharma and S. Prakash, "Optimizing Dead Mileage in Urban Bus Routes,” Journal of Transportation Engineering, Vol. 112, No. 1, 1986, pp. 121-129. doi:10.1061/(ASCE)0733-947X(1986)112:1(121)

[5] H. S. Kasana and K. D. Kumar, "An Efficient Algorithm for Multiobjective Transportation Problems," Asia-Pacific Journal of Operational Research, Vol. 17, 2000, pp. 27-40.

[6] A. K. Agrawal and S. L. Dhingra, “An Optimal Program for Augmentation of Capacities of Depots and Shipment of Buses from Depots to Starting Points of Routes," Indian Journal of Pure and Applied Mathematics, Vol. 20, No. 2, 1989, pp. 111-120.

[7] S. Prakash and V. Saini, "Selection of Optimal Site for New Depot of Specified Capacity with Two Objectives," Indian Journal of Pure and Applied Mathematics, Vol. 20, No. 5, 1989, pp. 425- 432.

[8] A. S. Pepin, G. Desaulniers and A. Hertz, "Comparison of Heuristic Approaches for the Multiple Depot Vehicle Scheduling Problem," Les Cahiers du GERAD, Vol. 65, 2006.

[9] C. B. Djiba, “Optimal Assignment of Routes to a Terminal for an Urban Transport Network. Master of Research Engineering Sciences," Cheikh Anta Diop University, 2008.

[10] Full Traffic of Dakar Dem Dikk (2008-2009), InputOutput File. http://www.demdikk.com

[11] IBM ILOG CPLEX Optimization Studio V12.3, Inc. Using the CPLEXR Callable Library and CPLEX Barrier and Mixed Integer Solver Options. 2011.

http://www-01.ibm.com/software/integration/optimizatio $\mathrm{n}$ /cplex-optimization-studio

[12] EMME User's Manual, 2007. 